\title{
Cytohesin-2 as a novel prognostic marker for hepatocellular carcinoma
}

\author{
KEDONG XU, JIE GAO, XUE YANG, YINGMIN YAO and QINGGUANG LIU \\ Department of Hepatobiliary Surgery, The First Hospital of Xi'an Jiaotong University, \\ College of Medicine, Xi'an, Shaanxi 710061, P.R. China
}

Received November 1, 2012; Accepted January 18, 2013

DOI: $10.3892 /$ or.2013.2366

\begin{abstract}
Cytohesin-2 is overexpressed in human lung cancer and it activates cytoplasmic ErbB receptors. Inhibition of cytohesin- 2 by SecinH3 reduces growth of EGFR-dependent lung cancer xenografts and improves the treatment of primarily EGFR-TKI-resistant lung cancers. Cytohesin-2 promotes HepG2 proliferation through the IGF pathway, and VEGF-dependent initiation of angiogenesis by regulation of VEGFR-2 internalization in endothelial cells, vessel permeability and ultimately endothelial proliferation. The purpose of this study was to evaluate the effects of cytohesin-2 in hepatocellular carcinoma (HCC) . In the current study, we collected $40 \mathrm{HCC}$ tissues and detected cytohesin-2 mRNA expression in the $40 \mathrm{HCC}$ tissues by using quantitative real-time polymerase chain reaction (qRT-PCR), as well as its protein expression by using immunohistochemistry and western blot analysis. We found that cytohesin-2 was more highly expressed in HCC compared to adjacent non-tumorous liver tissues, and cytohesin-2 expression was significantly increased in specimens with high $\alpha$-fetoprotein and vascular invasion. Both univariate and multivariate analyses indicated that there is an association between cytohesin-2 expression and overall survival (OS) and disease-free survival (DFS). Moreover, stratified analysis showed that patients in tumor-node-metastasis (TNM) stage I with higher cytohesin-2 levels had shorter OS and DFS than those with lower cytohesin-2 levels. In conclusion, cytohesin-2 may identify low-and high-risk individuals with HCC and may be a valuable indicator for stratifying prognosis of TNM stage I patients. Cytohesin-2 may serve as a novel prognostic biomarker for HCC.
\end{abstract}

\section{Introduction}

Hepatocellular carcinoma (HCC) is one of the most common solid tumors worldwide, particularly in East Asia and in

Correspondence to: Professor Qingguang Liu, Department of Hepatobiliary Surgery, The First Hospital of Xi'an Jiaotong University, College of Medicine, Xi'an, Shaanxi 710061, P.R. China

E-mail: liuqingguang@vip.sina.com

Key words: cytohesin-2, hepatocellular carcinoma, biomarker, prognosis
Sub-Saharan Africa $(1,2)$. It is the second most common cause of cancer-related mortality in men, and the sixth in women (3). Currently, the only curative therapeutic options for early-stage HCC are surgical interventions, including percutaneous ablation, hepatic resection, and liver transplantation. Fewer than $12 \%$ of diagnosed HCC patients are eligible for curative therapies in developing countries (4). Survival of HCC varies widely, and similar clinicopathological characteristics are likely attributable to heterogeneous biological behavior of tumor cells (5). Although recent studies have found some abnormal gene expressions in HCC that could serve as prognostic markers, knowledge of molecules that could help forecast early recurrence of HCC is limited.

Recently, Bill et al (6) found that cytohesin-2 was significantly overexpressed in human lung cancer, and inhibition of cytohesin could lead to reduction of lung cancer xenografts via activation of cytoplasmic ErbB receptors. Cytohesin-2 is part of the cytohesin family, which are guanine nucleotide exchange factors (GEFs) for ADP ribosylation factors (ARFs) that belong to the family of small Ras-like GTPases. As in the case of other small GTPases, ARF function critically depends on activation by GEFs (7). Thus, since ARFs are involved in controlling cytoskeletal dynamics, cell migration, vesicular traffic, and signaling $(8,9)$, cytohesins are key regulators of these processes. Cytohesin was initially reported in 1996 by Kolanus et al (10). However, recent studies have focused on physiological action, and the function of cytohesin in pathological states, particularly in cancer, is limited (11). Cytohesin critically affects cytoplasmic conformational activators of ErbB receptors, which are phosphoinositide 3-kinase (PI3K)/Akt/ mTOR pathway in HEK-293 cells. Recognized abnormalities in HCC include aberrant signaling through the PI3K/AKT and mTOR pathways (12). Cytohesin-2 overexpression in human lung cancer is reportedly correlated with auto-phosphorylation of EGFR (6); EGFR auto-phosphorylation was also identified in HCC (13). Lim et al (11) reported cytohesin-2 as critical to the insulin and insulin-like growth factor (IGF) pathway in HepG2 cells. Another study showed the role of cytohesin-2 in vascular endothelial growth factor (VEGF)-dependent initiation of angiogenesis by regulating VEGF Receptor (VEGFR)-2 internalization in endothelial cells (14).

In the present study, we examined the expression of cytohesin- 2 gene and protein in 80 samples of paired tumor and the surrounding non-tumorous liver tissue, and these samples 
were collected from 40 patients with HCC. The correlations between cytohesin-2 expression and the clinicopathological characteristics were evaluated, and the prognostic significance of cytohesin-2 in HCC was also elucidated.

\section{Materials and methods}

Patients and tissue specimens. The study group consisted of 40 HCC patients who underwent surgery (hepatic resection) at the First Hospital of Xi'an Jiaotong University, China (from 2008 to 2009). All tumors and surrounding non-tumorous liver tissues were collected at surgical resection and stored immediately at $-80^{\circ} \mathrm{C}$ until analysis. All specimens were confirmed histologically. Written informed consent, as required by the Institutional Review Board, was obtained from all patients. The clinicopathological profiles of the patients enrolled in the study were collected. The study was approved by the Local Medical Ethics Committee.

Antibodies. Polyclonal rabbit anti-cytohesin-2 (10405-1-AP) and polyclonal rabbit anti- $\beta$-actin (10497-1-AP) were obtained from Proteintech Group (Chicago, IL, USA). Goat anti-mouse and goat anti-rabbit $\operatorname{IgG}(\mathrm{H}+\mathrm{L})$, peroxidase conjugated were obtained from Pierce Biotechnology (USA).

RNA preparation and reverse transcription. Total RNA was extracted from the HCC and surrounding non-tumorous liver tissue samples with RNAfast200 (Fastagen Biotechnology, Shanghai, China). The amount of RNA was measured spectrophotometrically by absorbance at $260 \mathrm{~nm}$. First-strand cDNA was generated from RNA with the RevertAid First Strand cDNA Synthesis kit from Fermentas (Shanghai, China).

Quantitative real-time polymerase chain reaction ( $q R T-P C R)$. qRT-PCR was performed in a Bio-Rad Real-time iQ5 System (Bio-Rad, Philadelphia, PA, USA) using SYBR Premix Ex Taq II (Takara, Dalian, China). Thermocycling was carried out in a final volume of $20 \mu \mathrm{l}$ containing $1.0 \mu \mathrm{l}$ of the cDNA sample, $100 \mathrm{nM}$ each of the cytohesin- 2 or $\beta$-actin primers (forward and reverse), and $10 \mu 1$ of SYBR Premix Ex Taq II (including TaqDNA polymerase, reaction buffer, and deoxynucleotide triphosphate mixture). The primers for qRT-PCR were: cytohesin-2, forward, 5'-acggtgccatgactgaggtg-3 and reverse, $5^{\prime}$-tgctgaaggtcagtgtgacgtg-3'; $\beta$-actin, forward, 5 '-tgtccaccttccagcagatgtg-3' and reverse, $5^{\prime}$-agtcctcggccacattgtgaac-3' (all produced by Takara). The PCR amplification consisted of 40 cycles $\left(95^{\circ} \mathrm{C}\right.$ for $5 \mathrm{sec}, 55^{\circ} \mathrm{C}$ for $\left.30 \mathrm{sec}\right)$ after an initial denaturation step $\left(95^{\circ} \mathrm{C}\right.$ for $\left.10 \mathrm{sec}\right)$. To correct for differences in both quality and quantity between samples, $\beta$-actin was used as an internal control. The targets were obtained from the same mRNA preparations. Melting curves were performed to ensure only one product was amplified.

Cytohesin-2 mRNA expression score. The relative amount of cytohesin-2 in mRNA from HCC (T) and the surrounding non-tumorous liver tissues $(\mathrm{N})$ that were normalized to $\beta$-actin mRNA was calculated. The cytohesin- 2 mRNA expression score in each tissue was defined as $2^{-\Delta \Delta \mathrm{Ct}} ; \Delta \Delta \mathrm{Ct}=\left(\mathrm{Ct}_{\text {Cytohesin-2- }}\right.$ $\left.\mathrm{Ct}_{\beta \text {-actin }}\right)_{\mathrm{T}^{-}}\left(\mathrm{Ct}_{\mathrm{Cytohesin}-2}-\mathrm{Ct}_{\beta \text {-actin }}\right)_{\mathrm{N}}$. The expression score $<1$ was identified as low cytohesin-2 mRNA expression, and $>1$ was identified as high cytohesin-2 mRNA expression (there was no score $=1)(15)$.

Immunohistochemical assay (IHC). For IHC, fresh tissues were fixed in $10 \%$ neutral buffered formalin for $16 \mathrm{~h}$ at $4{ }^{\circ} \mathrm{C}$ and then placed in a Thermo Shandon tissue processor, and embedded in paraffin. Sections were heated in a $60^{\circ} \mathrm{C}$ oven, and wax removed by three changes of xylene, followed by a graded ethanol series $(100,95,90$ and $80 \%)$ before being subjected to a final wash in double-distilled $\mathrm{H}_{2} \mathrm{O}$. After quenching endogenous peroxidase activity with $3 \% \mathrm{H}_{2} \mathrm{O}_{2}$ for 10 min and blocking with BSA for $30 \mathrm{~min}$, sections were incubated at $4^{\circ} \mathrm{C}$ overnight with primary antibody against cytohesin-2 at a dilution of 1:100. Detection of cytohesin-2 was achieved with the Envision-horseradish peroxidase system (DakoCytomation, Glostrup, Denmark). All slides were counterstained with Gill's Hematoxylin for 1 min, dehydrated, and mounted for light microscopic evaluation independently by two experienced pathologists. A cutoff point of 25\% was used in our statistical analysis; sections were classified as negative, weak (0-25\%) or strong ( $\geq 25 \%)(16)$. The staining intensity and average percentage of positive liver cells were assayed for 10 independent high magnification (x400) fields. The total score was calculated by multiplying the staining intensity and the percentage of positive liver cells. Sections with a total score of $>1$ were defined as exhibiting positive staining for cytohesin-2 (17).

Protein preparation. Samples were homogenized in ice-cold RIPA buffer [1X phosphate-buffered saline (PBS), $1 \%$ Nonidet P-40, $0.5 \%$ sodium deoxycholate, $1 \%$ sodium dodecyl sulfate (SDS)] with protease inhibitors (Xianfeng Biotech, Xi'an China), then incubated on ice for $20 \mathrm{~min}$ and centrifuged at $12,000 \mathrm{rpm}$ for $15 \mathrm{~min}$ at $4^{\circ} \mathrm{C}$ to specification. The supernatant was quantified by Bradford BCA protein assay and stored at $-80^{\circ} \mathrm{C}$ until further use.

Western blot analysis. The protein samples $(20 \mu \mathrm{g})$ were separated by $10 \%$ SDS-PAGE; proteins were then transferred to polyvinylidene fluoride (PVDF) membranes which were blocked for $1 \mathrm{~h}$ with $1 \%$ BSA in Tris-buffered saline with Tween-20 (TBST) buffer (20 mM/l Tris, $\mathrm{pH} 7.6,100 \mathrm{mM} / 1$ $\mathrm{NaCl}, 0.1 \%$ Tween-20). This was followed by incubation with primary antibodies, including polyclonal antibody against cytohesin-2 (1:1,000 dilution) and $\beta$-actin (1:1,000 dilution) in TBST buffer containing $1 \% \mathrm{BSA}$, at $4^{\circ} \mathrm{C}$ overnight. After washing three times with TBST buffer, membranes were incubated with a horseradish peroxidase-conjugated goat anti-rabbit IgG and goat anti-rabbit IgG as secondary antibodies (1:10,000 dilution) for $1 \mathrm{~h}$ at room temperature. After the membranes were washed four times again in TBST buffer, reactions were visualized with the ECL detection system (Millipore, Billerica, MA, USA). Values for cytohesin- 2 were normalized to $\beta$-actin expression; cytohesin-2 protein values were compared between HCC and adjacent non-tumorous liver tissue. Ratios $>1$ were identified as higher cytohesin- 2 protein expression; ratios $\leq 1$ were identified as lower cytohesin-2 expression (18). All western blot analyses were repeated at least three times.

Follow-up. The follow-up duration was defined as the interval between the date of surgery and the date of death or last 

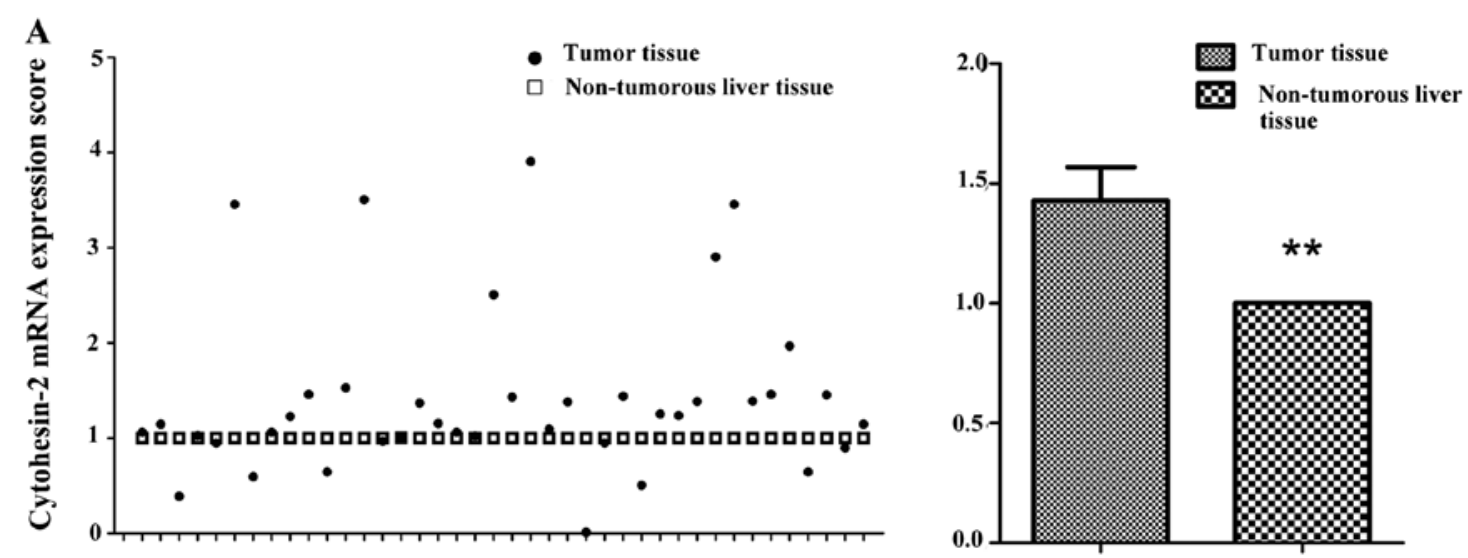

Tissue specimens

B
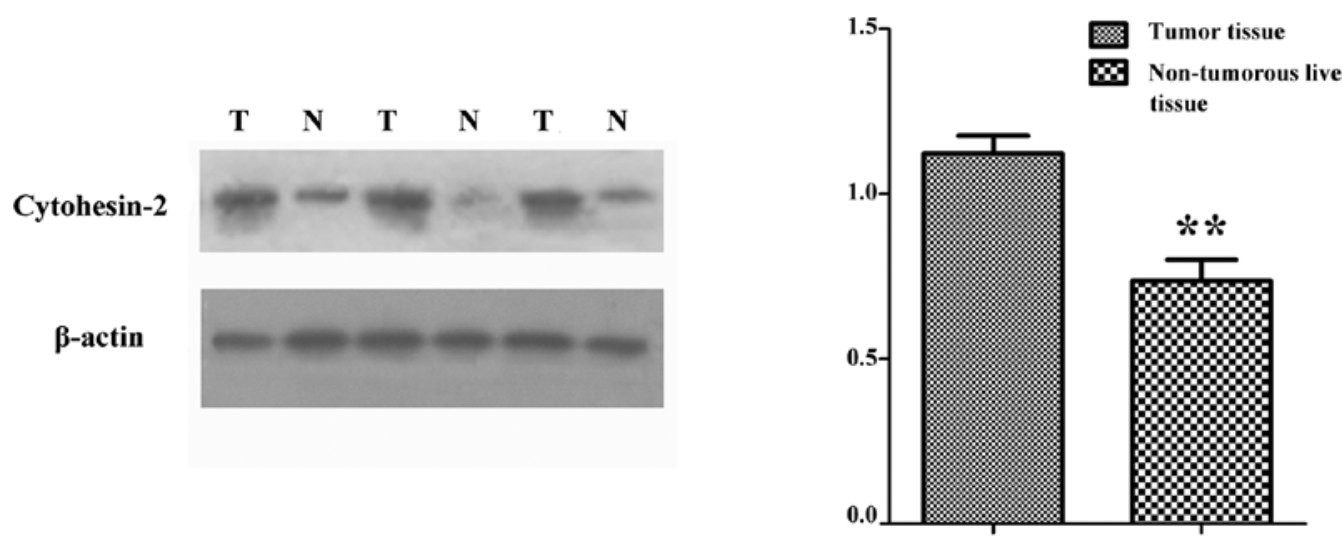

Figure 1. qRT-PCR and western blot analysis for cytohesin-2 expression. (A) Cytohesin-2 mRNA expression in HCC and the surrounding non-tumorous liver tissue. (B) Cytohesin-2 protein expression in HCC and the surrounding non-tumorous liver tissue. The results are normalized to $\beta$-actin. The data are expressed as the means \pm SEM. ${ }^{* *} \mathrm{P}<0.01$. HCC, hepatocellular carcinoma; $\mathrm{T}$, tumor tissue; $\mathrm{N}$, non-tumorous liver tissue.

follow-up. The study was censored on 31 January 2012. The median follow-up time was 25.32 months (range, 15-36). All patients were followed up every 1-3 months in the first year and every 3-6 months thereafter. The follow-up protocol included physical examination, serum $\alpha$-fetoprotein (AFP) level, chest X-ray, abdominal ultrasonography and computed tomography. Computed tomography and/or magnetic resonance imaging and/or positron emission tomography were performed when intrahepatic relapse or distant metastasis was suspected.

Statistical analysis. All statistical analyses were carried out using the SPSS 16.0 statistical software package (SPSS Inc., Chicago, IL, USA). The associations between cytohesin-2 expression and clinicopathological parameters were analyzed using Student's t-test. Overall and disease-free survival curves were generated using the Kaplan-Meier method; differences between curves were assessed by the log-rank test. Independent prognostic factors were estimated by the Cox proportional hazards stepwise regression model. All P-values were 2 -sided. $\mathrm{P}<0.05$ was considered to indicate statistically significant differences.

\section{Results}

Cytohesin-2 expression in HCC and surrounding non-tumorous liver tissue specimens. We first examined cytohesin-2 expression in $40 \mathrm{HCC}$ patient specimens and surrounding non-tumorous liver tissues, using qRT-PCR and western blot analysis (Fig. 1). Cytohesin-2 mRNA expression was between 0.13 and 3.97 (average $\pm \mathrm{SD}, 1.43 \pm 0.89$ ) in HCC tissues, which was higher than in surrounding non-tumorous liver tissues $(\mathrm{P}=0.005)$ (Table I). Cytohesin-2 protein expression was between 0.21 and 1.56 (average $\pm \mathrm{SD}, 1.12 \pm 0.33$ ) in HCC tissues, which was higher than in surrounding non-tumorous liver tissues (average $\pm \mathrm{SD}$, $0.70 \pm 0.39 ; \mathrm{P}=0.009$ ) (Table I and Fig. 1). Protein expression was consistent with that of mRNA in the same monitored samples (data not shown). Similar results were obtained from the same batch of HCC tissues using IHC, which indicated cytohesin-2 to be more highly expressed in tumors than in surrounding non-tumorous liver tissues. Cytohesin-2 was located in the cytoplasm (Fig. 2).

Correlation between cytohesin-2 expression and clinicopathological parameters. Subsequently, clinicopathological data were correlated with the cytohesin-2 expression. Cytohesin-2 protein expression was significantly associated with high AFP $(\mathrm{P}=0.01)$ (Table I and Fig. $3 \mathrm{~A})$ and vascular invasion $(\mathrm{P}=0.01)$ (Table I and Fig. 4A). The bivariate correlation showed AFP and cytohesin- 2 protein expression were positively correlated (correlation coefficient $=0.417, \mathrm{P}=0.01$ ). Similar results were observed when we checked cytohesin- 2 protein expression and vascular invasion (correlation coefficient $=0.361, \mathrm{P}=0.022$ ). There was no relationship between cytohesin-2 expression and other clinicopathological variables, including gender, age, 
Table I. Clinicopathological characteristics and cytohesin-2 expression in HCC.

\begin{tabular}{|c|c|c|c|c|c|c|}
\hline $\begin{array}{l}\text { Clinicopathological } \\
\text { characteristic }\end{array}$ & Variable & $\begin{array}{l}\text { No. of } \\
\text { cases }\end{array}$ & $\begin{array}{l}\text { Cytohesin-2 mRNA } \\
\text { expression scores } \\
\text { (means } \pm \mathrm{SD})\end{array}$ & P-value & 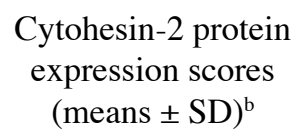 & P-value \\
\hline \multirow[t]{2}{*}{ Gender } & Male & 31 & $1.50 \pm 0.96$ & \multirow[t]{2}{*}{0.345} & $1.42 \pm 0.88$ & \multirow[t]{2}{*}{0.408} \\
\hline & Female & 9 & $1.18 \pm 0.49$ & & $1.16 \pm 0.46$ & \\
\hline \multirow[t]{2}{*}{ Age (years) } & $>60$ & 10 & $1.67 \pm 0.88$ & \multirow[t]{2}{*}{0.32} & $1.30 \pm 0.84$ & \multirow[t]{2}{*}{0.412} \\
\hline & $\leq 60$ & 30 & $1.35 \pm 0.89$ & & $1.55 \pm 0.74$ & \\
\hline \multirow[t]{2}{*}{ Background liver status } & With cirrhosis & 37 & $1.44 \pm 0.90$ & \multirow[t]{2}{*}{0.712} & $1.38 \pm 0.83$ & \multirow[t]{2}{*}{0.702} \\
\hline & Without cirrhosis & 3 & $1.24 \pm 0.73$ & & $1.19 \pm 0.59$ & \\
\hline \multirow[t]{2}{*}{ Maximal tumor size (mm) } & $<50$ & 28 & $1.46 \pm 0.94$ & \multirow[t]{2}{*}{0.757} & $1.39 \pm 0.87$ & \multirow[t]{2}{*}{0.753} \\
\hline & $>50$ & 12 & $1.36 \pm 0.78$ & & $1.30 \pm 0.68$ & \\
\hline \multirow[t]{2}{*}{$\alpha$-Fetoprotein (ng/ml) } & $\leq 400$ & 20 & $1.06 \pm 0.38$ & \multirow[t]{2}{*}{$0.007^{\mathrm{a}}$} & $1.04 \pm 0.35$ & \multirow[t]{2}{*}{$0.01^{\mathrm{a}}$} \\
\hline & $>400$ & 20 & $1.79 \pm 1.09$ & & $1.69 \pm 1.00$ & \\
\hline \multirow[t]{2}{*}{ Tumor number } & Single & 33 & $1.39 \pm 0.88$ & \multirow[t]{2}{*}{0.593} & $1.33 \pm 0.82$ & \multirow[t]{2}{*}{0.511} \\
\hline & Multiple & 7 & $1.59 \pm 0.93$ & & $1.55 \pm 0.78$ & \\
\hline \multirow[t]{2}{*}{ Histology } & Well and Mod & 24 & $1.49 \pm 0.96$ & \multirow[t]{2}{*}{0.621} & $1.40 \pm 0.82$ & \multirow[t]{2}{*}{0.771} \\
\hline & Poor & 16 & $1.34 \pm 0.78$ & & $1.32 \pm 0.82$ & \\
\hline \multirow[t]{2}{*}{ Capsule formation } & + & 27 & $1.55 \pm 1.02$ & \multirow[t]{2}{*}{0.203} & $1.49 \pm 0.94$ & \multirow[t]{2}{*}{0.174} \\
\hline & - & 13 & $1.17 \pm 0.40$ & & $1.11 \pm 0.36$ & \\
\hline \multirow[t]{2}{*}{ Vascular invasion } & + & 9 & $2.26 \pm 1.26$ & \multirow[t]{2}{*}{$0.001^{\mathrm{a}}$} & $2.09 \pm 1.19$ & \multirow[t]{2}{*}{$0.01^{\mathrm{a}}$} \\
\hline & - & 31 & $1.18 \pm 0.56$ & & $1.15 \pm 0.52$ & \\
\hline \multirow[t]{2}{*}{ TNM stage } & I & 30 & $1.52 \pm 0.98$ & \multirow[t]{2}{*}{0.241} & $1.45 \pm 0.89$ & \multirow[t]{2}{*}{0.239} \\
\hline & II, III, IV & 10 & $1.14 \pm 0.45$ & & $1.10 \pm 0.41$ & \\
\hline
\end{tabular}

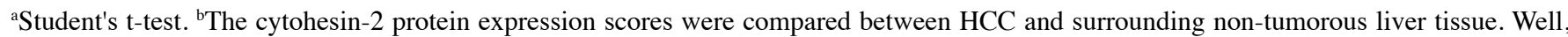
well-differentiated HCC; mod, moderately differentiated HCC; poor, poorly differentiated HCC. HCC, hepatocellular carcinoma.
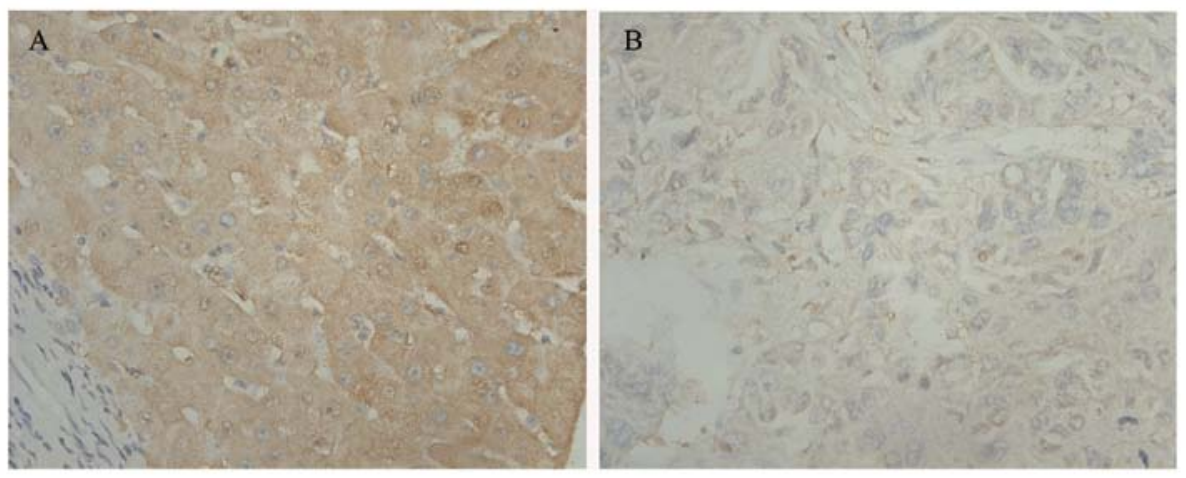

Figure 2. Immunohistochemical assay for cytohesin-2 in HCC and the surrounding non-tumorous liver tissue. (A) Cytohesin-2 protein expression in HCC (x40). (B) Cytohesin-2 protein expression in the surrounding non-tumorous liver tissue (x40). Cytohesin-2 protein expression (x40) was higher in HCC than in the surrounding non-tumorous liver tissue. HCC, hepatocellular carcinoma.

tumor size, liver cirrhosis, TNM stage, tumor number, tumor capsule, and Edmondson-Steiner grade. All patients were infected with hepatitis B virus, as hepatitis B surface antigen (HBsAg) status was not mentioned. Cytohesin-2 mRNA expression was also associated with high AFP level $(\mathrm{P}=0.007)$ (Table I and Fig. 3B) and vascular invasion $(\mathrm{P}=0.001)$ (Table I and Fig. 4B).
Prognostic of HCC subtypes defined by cytohesin-2 level. During the course of follow-up, 30/40 patients (75.0\%) were found with intrahepatic recurrence, 4 patients $(10 \%)$ developed distant metastases. Twenty-four patients $(60 \%)$ succumbed to cancer-related causes, 8 patients $(20 \%)$ succumbed to liver cirrhosis-related diseases (such as hepatic failure and upper gastrointestinal hemorrhage), 3 patients (7.5\%) succumbed 
Table II. Multivariate analysis of factors contributing to overall survival and disease-free survival in HCC patients.

\begin{tabular}{|c|c|c|c|c|}
\hline \multirow[b]{2}{*}{ Variable } & \multicolumn{2}{|c|}{ Overall survival } & \multicolumn{2}{|c|}{ Disease-free survival } \\
\hline & HR $(95 \%$ CI $)$ & P-value & HR $(95 \%$ CI $)$ & P-value \\
\hline Tumor size & $1.375(1.158-1.892)$ & 0.026 & $2.911(1.203-7.043)$ & 0.018 \\
\hline Tumor number & $2.921(0.958-8.910)$ & 0.060 & $3.613(1.112-11.733)$ & 0.033 \\
\hline Histology & $5.499(1.897-15.938)$ & 0.002 & $2.498(0.996-6.267)$ & 0.051 \\
\hline Vascular invasion & $3.560(1.449-8.571)$ & 0.006 & $4.044(1.270-12.882)$ & 0.018 \\
\hline Cytohesin-2 protein expression level & $3.251(1.338-7.902)$ & 0.009 & $5.558(1.897-16.284)$ & 0.002 \\
\hline
\end{tabular}

HCC, hepatocellular carcinoma; HR, hazard ratio; CI, confidence interval.
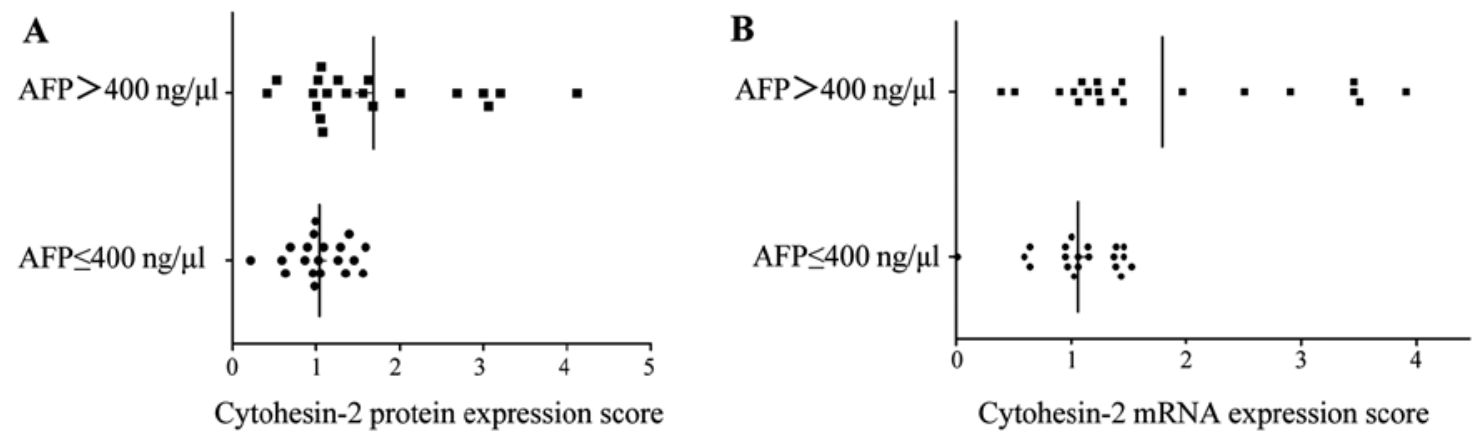

Figure 3. Correlation between cytohesin-2 expression and AFP level. (A) Cytohesin-2 protein expression scores in cases with different AFP levels. (B) Cytohesin-2 mRNA expression scores in cases with different AFP levels. A significant increase in cytohesin-2 expression scores was observed in cases with higher AFP level.

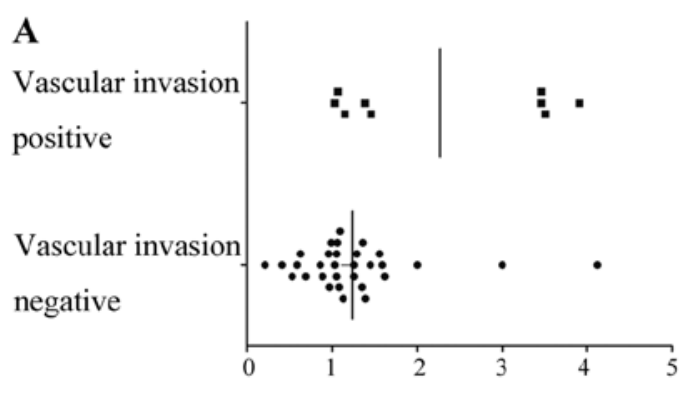

Cytohesin-2 protein expression score

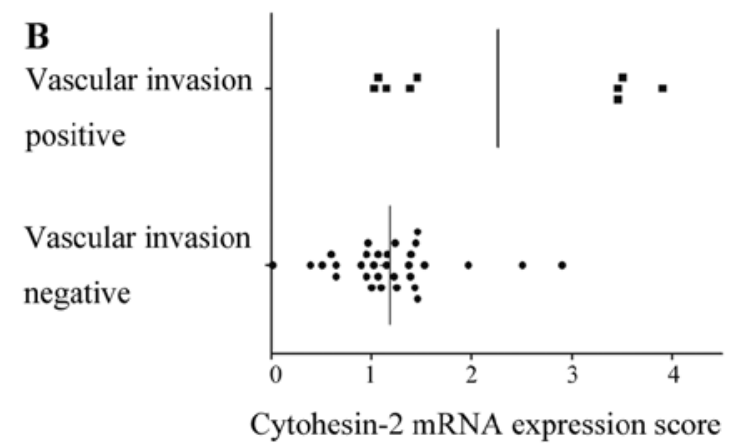

Cytohesin-2 mRNA expression score

Figure 4. Correlation between cytohesin-2 expression and vascular invasion. (A) Cytohesin-2 protein expression scores in cases with or without vascular invasion. (B) Cytohesin-2 mRNA expression scores in cases with or without vascular invasion. A significant increase in cytohesin-2 expression scores was observed in cases with vascular invasion compared to those without vascular invasion.

to diseases unrelated to cancer, and 5 patients (12.5\%) were still alive. We selected gender, age, tumor size, liver cirrhosis, TNM stage, tumor number, tumor capsule, and EdmondsonSteiner grade, cytohesin-2 as prognostic factors for the analysis of overall survival (OS) and disease-free survival (DFS). Significant OS and DFS advantages were observed in patients with low cytohesin-2 protein expression. Median survival was 31.72 months (24-36) of the low-level group, which was significantly longer than that of the high-level group (23.19 months) (15-36; $\mathrm{P}=0.005)$. DFS was 21.40 months $(9-29)$ in the lowlevel group, which was significantly longer than that of the high-level group (10.17 months) $(6-19 ; \mathrm{P}<0.01)$.
Stratified univariate and multivariate analysis. In a univariate analysis model, cytohesin- 2 mRNA and protein expression were significantly associated with $\mathrm{OS}(\mathrm{P}=0.022 ; \mathrm{P}=0.048$, respectively) and DFS ( $\mathrm{P}<0.01 ; \mathrm{P}=0.001$, respectively) (Fig. 5). The similar result was obtained when we performed multivariate analysis, and it showed that cytohesin-2 protein expression level was associated with OS $(\mathrm{P}=0.009)$ and DFS $(\mathrm{P}=0.002)$ (Table II). Cytohesin-2 mRNA expression level as a factor for multivariate analysis was then examined, and we found the similar result as cytohesin-2 protein expression level as a factor we showed in Table II. Since survival may be associated with TNM stage, we stratified the data according to TNM stage 

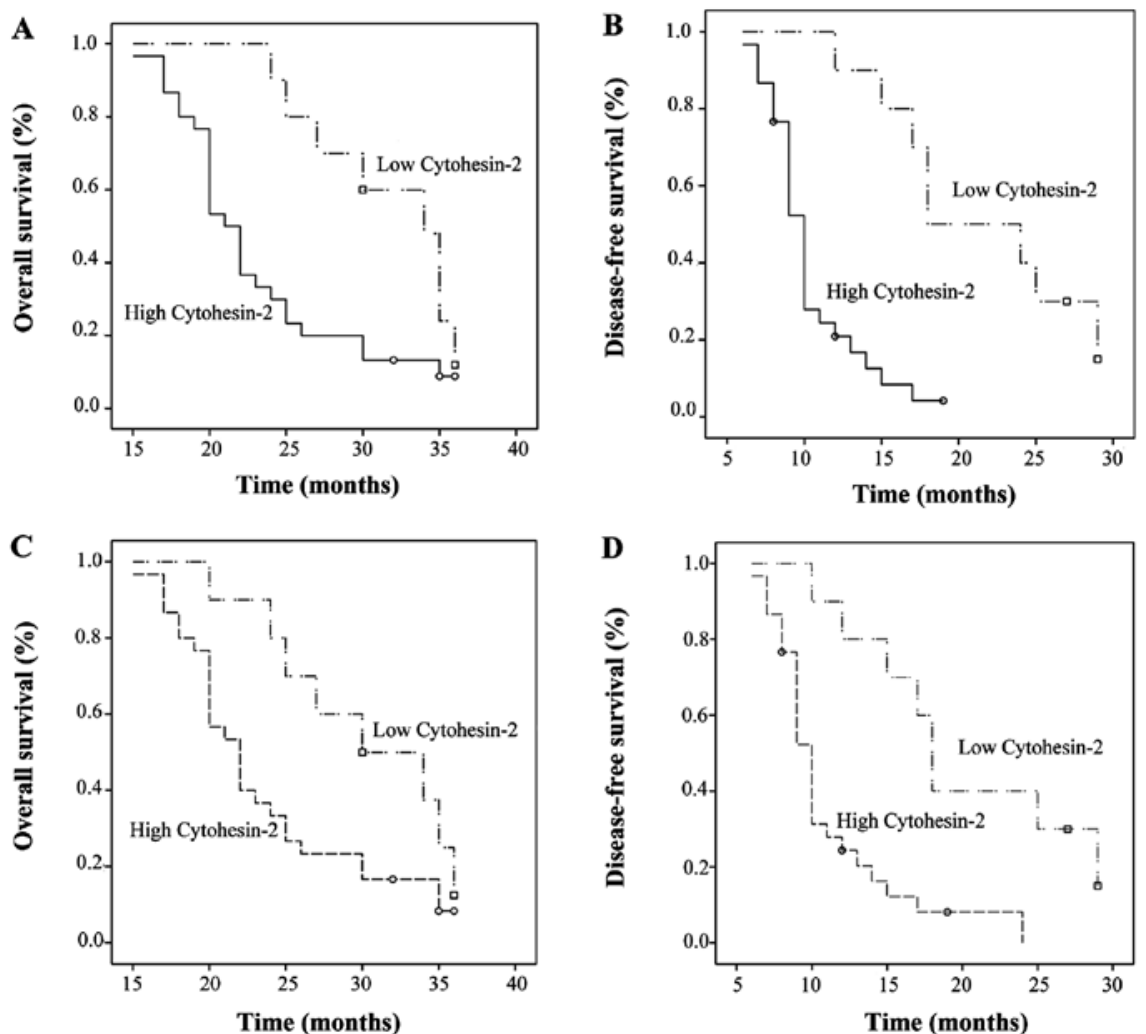

Figure 5. Kaplan-Meier survival curves according to cytohesin-2 expression in HCC patients. (A) Overall survival (OS) for cytohesin-2 mRNA expression (log-rank $\mathrm{P}<0.05$ ). (B) Disease-free survival (DFS) for cytohesin-2 mRNA expression (log-rank $\mathrm{P}<0.01)$. (C) OS for cytohesin-2 protein expression $(\log -\mathrm{rank}$ $\mathrm{P}<0.05)$. (D) DFS for cytohesin-2 protein expression (log-rank $\mathrm{P}<0.001)$.
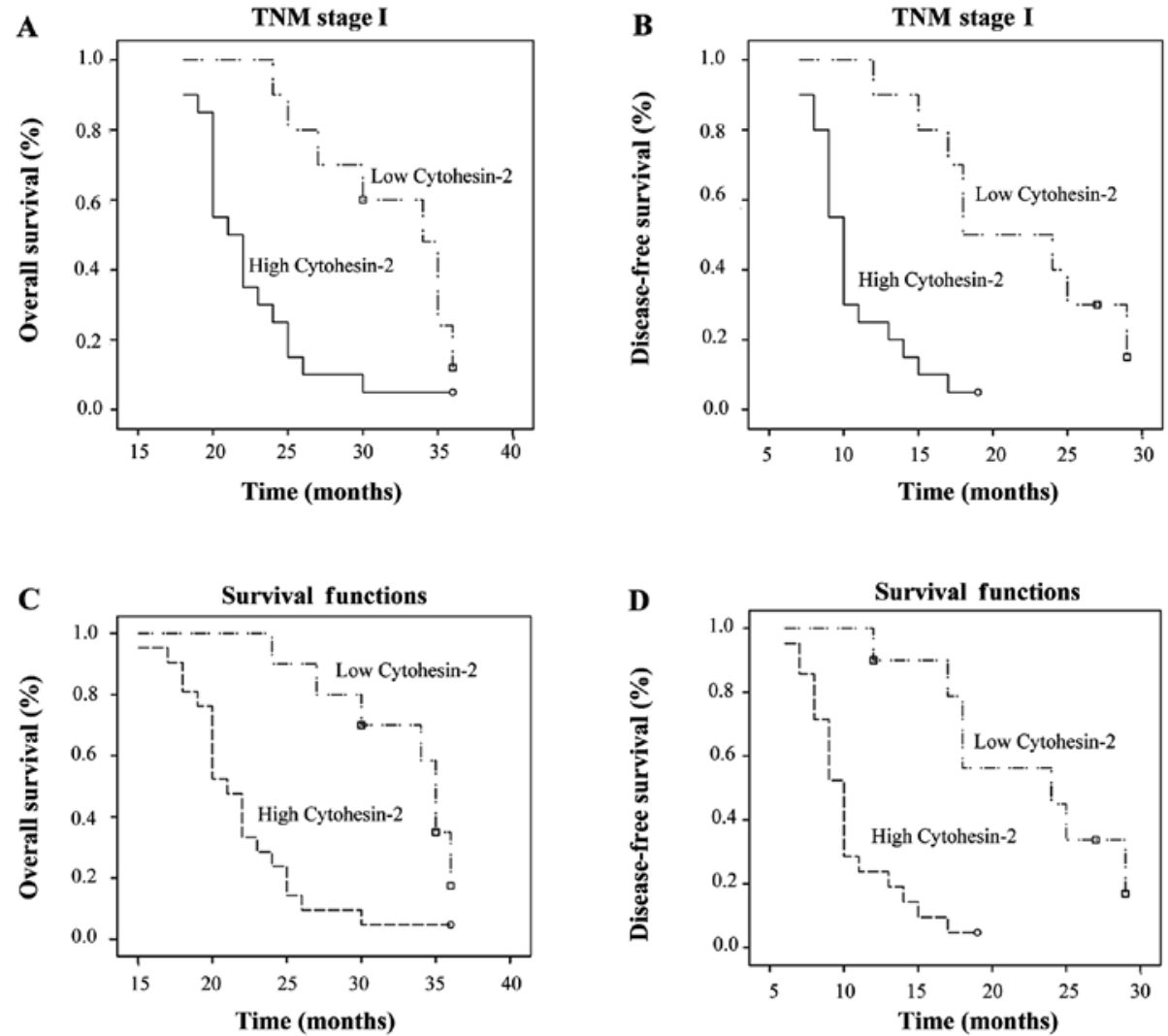

Figure 6. Kaplan-Meier curves according to cytohesin-2 expression in TNM stage I HCC patients. (A) Overall survival (OS) for cytohesin-2 mRNA expression (log-rank $\mathrm{P}<0.05$ ) (B) Disease-free survival (DFS) for cytohesin-2 mRNA expression (log-rank $\mathrm{P}<0.01)$. (C) OS for cytohesin-2 protein expression $(\log -\mathrm{rank}$ $\mathrm{P}<0.01)$. (D) DFS for cytohesin-2 protein expression (log-rank $\mathrm{P}<0.01$ ). 
and investigated the prognostic value of cytohesin- 2 for TNM stage I patients; there were no significant differences in the patient background profiles. For the 30 TNM stage I patients, significant correlations were found between cytohesin-2 expression and OS and DFS. Cytohesin-2 was an independent prognostic factor for survival in TNM stage I patients. Patients with high cytohesin-2 mRNA and protein expression had poorer OS $(\mathrm{P}=0.015 ; \mathrm{P}<0.01)$ and DFS $(\mathrm{P}<0.01 ; \mathrm{P}<0.01)$ compared to those with low cytohesin-2 expression in TNM stage I (Fig. 6).

\section{Discussion}

$\mathrm{HCC}$ is one of the most common solid tumors in mainland China, with an annual incidence of 24 per 100,000 people. The mortality rate of $\mathrm{HCC}$ is as high as its morbidity rate (19), due to its high recurrence rate which is as high as $54 \%$ at 5 years, even for early-stage HCC treated with radical resection (20). Molecular markers for HCC recurrence are limited in earlystage disease, although some molecules have been identified as prognostic markers. For this reason, identifying genetic alterations that allow estimation of early $\mathrm{HCC}$ recurrence are important.

Cytohesin-2 is reported to be overexpressed in human lung cancer (6), and to participate in the IGF pathway in HepG2 cells (11). However, to our knowledge, cytohesin-2 expression in HCC has yet to be reported. In this study, we analyzed cytohesin-2 mRNA and protein expressions in $40 \mathrm{HCC}$ patients and correlated them with clinicopathological characteristics and prognoses to determine whether this biomarker could predict disease outcome. Cytohesin-2 expression in HCC tissue was significantly higher than in surrounding non-tumorous tissue, as Bill et al (6) found in human lung cancer. Markedly, high cytohesin-2 expression was significantly correlated with more aggressive cancer, in terms of shorter OS and DFS, AFP and vascular invasion, which are putative clinicopathological markers for HCC development, invasiveness and unfavorable prognosis (21). These data indicate that high cytohesin-2 expression occurs in HCC and is associated with an aggressive invasion phenotype, and that cytohesin-2 expression correlated with AFP which is a biomarker for proliferation (22). This finding is in agreement with previous studies; for example, Lim et al (11) reported that cytohesin-2 is involved in insulin and IGF pathways and promotes HepG2 proliferation. Cytohesin-2 promotes tumor proliferation and this function was identified in human lung cancer as SecinH3 which targets the Sec7 domain of the cytohesins (23) and reduces the growth of EGFR-dependent lung tumor xenografts (6). Cytohesin-2 expression correlated with vascular invasion; this was in accordance with the finding of Mannell et al (14) that cytohesin-2 affects VEGF-dependent initiation of angiogenesis by regulating VEGFR-2 internalization in endothelial cells. Cytohesin-2 scores in HCC with vascular invasion were used to separate samples into two groups. No statistical difference in intensity of vascular invasion was found between the high- and low-score groups. Furthermore, in the low-score group, there was one case in which a portal vein tumor thrombus (PVTT) protruded into the first main portal vein branch beyond the resection line for $>1 \mathrm{~cm}$ (19), and the others were located in the hepatic resec- tion area or protruded into the first main portal vein branch beyond the resection line for $<1 \mathrm{~cm}$. Nevertheless, in the highscore group, two cases of PVTT protruded into the first main portal vein branch beyond the resection line for $>1 \mathrm{~cm}$, and the PVTT extended into the main portal vein in one case. The lack of statistical difference may be due to the small number of samples, as patients with both HCC and PTVV have diminished access to hepatectomy. The effect of cytohesin-2 in angiogenesis is a future research priority for us.

Clinical stage is the most important factor in the prognosis of HCC patients. The International Union Against Cancer's TNM staging system is the most widely used. However, it is difficult for a surgeon to predict which individual TNM stage I patients will suffer early recurrence following curative treatment. Although several molecular markers have been shown to possess potential predictive significance, biomarkers that could identify patients with TNM stage I HCC who are likely to respond optimistically to curative excision remain substantially limited. Our stratified analysis showed that cytohesin-2 expression had clear prognostic value for OS and DFS in these patients, indicating that cytohesin- 2 could be used as a predictive tool to identify patients with TNM stage I HCC at high risk of recurrence.

High cytohesin-2 expression in HCC tissues predicts poor prognosis, which suggests that cytohesin-2 affects the HCC malignancy process. This information could identify high-risk HCC patients who may benefit from more intensive treatment and follow-up care following resection of primary tumors. Further studies are required to gain insight into the underlying biology of cytohesin-2 in HCC, and to develop new therapies targeted at cytohesin-2.

\section{Acknowledgements}

The authors thank Dr Ting Lei and Professor Gui-Hua Zhuang for the histologic confirmation of specimens and statistical analyses. This study was completed in the Key Laboratory of Forensic Medicine of the Ministry of Health of China, Xi'an Jiaotong University. The authors thank all the patients who participated in this study.

\section{References}

1. El-Serag HB and Rudolph L: Hepatocellular carcinoma: epidemiology and molecular carcinogenesis. Gastroenterology 132: 2557-2576, 2007.

2. Parkin DM, Bray F, Ferlay J and Pisani P: Global cancer statistics, 2002. Ca Cancer J Clin 55: 74-108, 2005.

3. Jemal A, Bray F, Center MM, Ferlay J, Ward E and Forman D: Global cancer statistics. Ca Cancer J Clin 61: 69-90, 2011

4. Kitisin K, Pishvaian MJ, Johnson LB and Mishra L: Liver stem cells and molecular signaling pathways in hepatocellular carcinoma. Gastrointest Cancer Res 1 (Suppl 2): S13-S21, 2007.

5. Dragani TA: Risk of HCC: Genetic heterogeneity and complex genetics. J Hepatol 52: 252-257, 2010.

6. Bill A, Schmitz A, Albertoni B, et al: Cytohesins are cytoplasmic ErbB receptor activators. Cell 143: 201-211, 2010.

7. Bos JL, Rehmann H and Wittinghofer A: GEFs and GAPs: Critical elements in the control of small G proteins. Cell 129: 865-877, 2007.

8. Casanova JE: Regulation of arf activation: the sec7 family of guanine nucleotide exchange factors. Traffic 8: 1476-1485, 2007.

9. Kolanus W: Guanine nucleotide exchange factors of the cytohesin family and their roles in signal transduction. Immunol Rev 218: 102-113, 2007. 
10. Kolanus W, Nagel W, Schiller B, et al: Alpha L beta 2 integrin/ LFA-1 binding to ICAM-1 induced by cytohesin-1, a cytoplasmic regulatory molecule. Cell 86: 233-242, 1996.

11. Lim J, Zhou M, Veenstra TD and Morrison DK: The CNK1 scaffold binds cytohesins and promotes insulin pathway signaling. Genes Dev 24: 1496-1506, 2010.

12. Thomas M: Molecular targeted therapy for hepatocellular carcinoma. J Gastroenterol 44: 136-141, 2009.

13. Kannangai R, Sahin F and Torbenson MS: EGFR is phosphorylated at Ty845 in hepatocellular carcinoma. Mod Pathol 19: 1456-1461, 2006.

14. Mannell HK, Pircher J, Chaudhry DI, et al: ARNO regulates VEGF-dependent tissue responses by stabilizing endothelial VEGFR-2 surface expression. Cardiovasc Res 93: 111-119, 2012.

15. Shirahata A, Fan W, Sakuraba K, et al: MACC 1 as a marker for vascular invasive hepatocellular carcinoma. Anticancer Res 31: 777-780, 2011.

16. Wang L, Chen S, Zhang M, et al: Legumain: A biomarker for diagnosis and prognosis of human ovarian cancer. J Cell Biochem 113: 2679-2686, 2012.

17. Tu K, Zheng X, Zan X, Han S, Yao Y and Liu Q: Evaluation of Fbxw7 expression and its correlation with the expression of c-Myc, cyclin E and p53 in human hepatocellular carcinoma. Hepatol Res 42: 904-910, 2012.
18. Tan NS, Michalik L, Di-Poi N, et al: Essential role of Smad3 in the inhibition of inflammation-induced PPAR beta/delta expression. EMBO J 23: 4211-4221, 2004.

19. Chen XP, Qiu FZ, Wu ZD, et al: Effects of location and extension of portal vein tumor thrombus on long-term outcomes of surgical treatment for hepatocellular carcinoma. Ann Surg Oncol 13: 940-946, 2006.

20. Cherqui D, Laurent A, Mocellin N, et al: Liver resection for transplantable hepatocellular carcinoma: long-term survival and role of secondary liver transplantation. Ann Surg 250: 738-746, 2009.

21. Cammà C, Schepis F, Orlando A, et al: Transarterial chemoembolization for unresectable hepatocellular carcinoma: meta-analysis of randomized controlled trials. Radiology 224: 47-54, 2002.

22. Fujioka M, Nakashima $Y$, Nakashima $O$ and Kojiro $M$ : Immunohistologic study on the expressions of $\alpha$-fetoprotein and protein induced by vitamin $\mathrm{K}$ absence or antagonist II in surgically resected small hepatocellular carcinoma. Hepatology 34: 1128-1134, 2001

23. Hafner M, Schmitz A, Grüne I, et al: Inhibition of cytohesins by SecinH3 leads to hepatic insulin resistance. Nature 444: 941-944, 2006. 